\title{
Desarrollo e integración de los niños con discapacidad visual a través deunas jornadas lúdico-deportivo-recreativas Development and integration of sighted disable children through a playful sports and recreational meetings
}

\author{
Manuel Gómez López*,Alfonso Valero Valenzuela*, Héctor Gutiérrez Barón** \\ * Universidad de Murcia y ** Universidad de Almería
}

\begin{abstract}
Resumen: Son numerosos los argumentos que posicionan la actividad física como un elemento indispensable para mejorar la calidad de vida y el mantenimiento de la salud, pero más aún entre los discapacitados que sufren una vida más sedentaria, faltos de estimulación e inseguros, en una sociedad que aún ha de adquirir una mayor concienciación. Desde este trabajo se plasma la experiencia práctica de unas jornadas lúdico-deportivo-recreativas con discapacitados visuales con el claro objetivo de desarrollar sus capacidades motrices, cognitivas, afectivas y sociales, así como la de sensibilizar al resto de la población sobre los problemas y dificultades con las que se encuentran en su vida diaria. Destacar la grata experiencia vivida tanto por los monitores, familiares como por los propios niños con la realización de las actividades propuestas, así como la necesidad de seguir promocionándolas con mayor asiduidad y en un mayor número de municipios.
\end{abstract}

Palabras clave: Discapacidad visual Actividades deportivas. Integración. Diversidad.

\begin{abstract}
There are a lot of arguments that place physical activity like a essential key to improve the life quality and health maintenance, however it is even more important in disable people who has a undergo sedentary life, with no help to practice exercise and insecure in a society still needs a greater awareness of it. This paper describes a practical experience of playful sport recreational meetings with sighted disable people trying to develop motor, cognitive, affective and social skills, as well as to make aware to the rest of population of the problems and difficulties which are in daily life. We emphasize the pleasure experience lived by instructors, relatives and children who made the activities, as well as the need to forest more frequently and in a greater number of municipalities.
\end{abstract}

Key words: Sighted disable. Sports activities. Integration. Diversity.

\section{Introducción.}

A lo largo de los últimos años se han ido sumando numerosos argumentos que posicionan la actividad física como un elemento indispensable para mejorar la calidad de vida y el mantenimiento de la salud (Gómez, 2005; Ruiz Juan y García Montes, 2005; Ruiz Juan y cols. 2005), pero ¿qué sucede con la actividad física en las personas con discapacidad o en el caso que nos atañe con discapacidades visuales?

La mayoría de los estudios estiman que entre el diez y el quince por ciento de la población española presenta algún tipo de discapacidad, aproximadamente unas 480.000 personas menores de 65 años (Cuadrado y cols., 2001).

Sobre un $80 \%$ de la información que adquirimos del entorno y que se necesita para el desarrollo de la vida cotidiana se obtiene a través del órgano de la visión, lo que significa que la mayoría de la información que se posee, las habilidades que se tienen y las actividades que se realizan han tenido como elemento básico de aprendizaje la visión (Barrado, 2004).

Entendiendo por discapacitados visuales al colectivo de personas ciegas, así como aquellas queúnicamente tienen una ligera percepción de la luz, nunca se nos debe olvidar que por ello, no dejan de ser sujetos con pleno derecho al uso y disfrute de la educación, al empleo, a la salud, al ocio, la cultura y al deporte (Valero y Gómez, 2005).

La gran mayoría de las personas con problemas visuales, no son ciegos, sino deficientes visuales con una gran variedad de patologías que limitan la visión y, por tanto, la percepción de muy diferentes formas. Estas personas pueden tener baja visión debido a la disminución de la agudeza visual, es decir, que no captan bien los contornos de las cosas y para poder verlas bien necesitan acercarse a ellas o utilizar gafas o elementos especiales (Fernández y cols., 2002a).

Sin embargo, son numerosos los motivos y necesidades que fundamentan aún más la práctica deportiva en este colectivo como manifiestan Linares (1997) y Loeckemeyer (2006) entre otros autores, al señalar

Fecha de recepción: 03-12-06 - Fecha de aceptación: 20-01-07

Correspondencia: Manuel Gómez López

C/ Valle Inclán, 10, $3^{\circ} 2$

04006 Almería

E-mail: mgomezlop@um.es que por su dificultad para desarrollar una movilidad independiente tienen una vida más sedentaria, mayores problemas traumatológicos debidos a malos hábitos posturales, menor nivel de coordinación por falta de estimulación visual al movimiento en los primeros años de vida e inseguridad en la realización de gestos motrices por la ausencia de vista.

Cuadrado y cols. (2001) realizaron un estudio con personas afectadas con una paraplejía, los cuales dependen de los miembros superiores de su cuerpo para poder desenvolverse en la mayoría de las actividades diarias y poseen una reducida movilidad física lo que les proporciona un bajo nivel de condición física, incrementándose el riesgo de padecer, entre otras muchas patologías, deficiencias cardiovasculares, músculoesqueléticas y obesidad. Mediante este trabajo se demostró que la práctica de actividad física basada en un programa de entrenamiento físico, mejora el nivel de su condición física, consiguiendo así una mayor independencia y autonomía en todas sus tareas cotidianas y en definitiva una mejora de la calidad de vida.

En el ámbito educativo, la diversidad se manifiesta tanto en función de factores sociales, culturales, geográficos, económicos, étnicos, religiosos, sexuales, etc., como de las propias capacidades del sujeto, es decir, intelectuales, motrices o sensoriales, por lo que para atender a esta diversidad, debemos actuar en la compensación o potenciación de aquellos factores que originan las situaciones de desventaja respecto al resto del grupo (Arráez, 1998), por lo que el objetivo de la Educación Física debe ser hacer al sujeto consciente de su propia realidad corporal, de sus posibilidades de actuación en el entorno físico y humano y de sus propias limitaciones. Para ello, el juego motor es un valioso instrumento de aprendizaje aprovechado como recurso para potenciar la formación integral del alumno con el objetivo de enriquecer cuantitativa y cualitativamente la motricidad del niño (Ríos, 2003).

Uno de los mayores problemas a los que se enfrentan los discapacitados visuales es el de la percepción, estructuración y organización del espacio, así como de los objetos situados en el mismo; no poseen un adecuado control dinámico de su propio cuerpo, debido a la falta de seguridad que la carencia de visión les produce, por lo que para determinadas actividades en desplazamientos es necesario que un compañero o el propio profesor le guíen verbalmente o incluso cogiéndole de la mano (Arráez, 1998). 
Otro de los grandes problemas, es que son incapaces de ver las formas expresivas corporales, lo que plantea serias dificultades a la hora de ejecutar gestos tanto en la vida diaria como en la práctica de actividades lúdico-deportivas, por lo que es necesario entrenarlos partiendo de la percepción táctil y la ejecución de gestos de lo analítico a lo global (Arráez, 1998).

Estos argumentos son más que suficientes para que los profesionales en el campo de la actividad físico deportiva y recreativa presten atención a este amplio sector de la sociedad y se planteen objetivos tales como promover el crecimiento, desarrollo y bienestar del individuo e incremento de su calidad de vida, a través de la provisión y satisfacción de necesidades, para disfrutar de los derechos que todos los individuos tienen en esencia por su condición, como es el derecho a la recreación. Un objetivo no menos importante y dirigido al resto de la población, ha de ser la sensibilización a los demás sobre los problemas y dificultades que tiene la realización de actividad física en tales condiciones (Ortiz, 2001).

La recreación es un derecho básico que contribuye con el mejoramiento de la calidad de vida individual y colectiva, que en el caso de las personas con limitación visual, se ha de orientar a la consecución de una verdadera integración funcional, en donde la persona experimenta una verdadera inclusión participativa en su comunidad y en su entorno en general y así favorece la interacción de la persona con el medio (Duque y Mosquera, 2004). Además, en las personas con limitación visual, la recreación se convierte en un instrumento facilitador de procesos de integración, debido a que esta población presenta grandes espacios para el tiempo libre, debiéndose promover acciones que potencien su uso para un esparcimiento enriquecedor.

Así, estudios como el realizado por Santana y Guillén (2002), tras analizar los intereses y actitudes hacia la actividad físico-deportiva existente en una muestra de 130 personas ciegas o con alguna deficiencia visual, reflejó el gran interés y nivel de práctica deportiva.

Según Barrado (2004), la práctica deportiva de estas personas, además de ser equivalente al resto de la población en lo que se refiere al desarrollo integral, es un elemento clave para avanzar en aspectos de la vida diaria tales como la autonomía personal, la autoestima y las relaciones sociales. Una de las características específicas de las personas ciegas y deficientes visuales es la disminuida coordinación y la pérdida de orientación en muchos casos incrementada por la sobreprotección paterna en los primeros años de la infancia y la carencia de movimientos elementales provocados por la misma.

La práctica de cualquier actividad físico-deportiva provoca la estimulación de muchos mecanismos musculares que no son utilizados a diario, desarrollando así las capacidades físicas a niveles parecidos para los que estamos diseñados genéticamente(Fernándezy cols., 2002a).

Siguiendo a López Rodríguez y Moreno Murcia (2000), consideramos que desde la práctica lúdica, deportiva y recreativa se ha de buscar la integralidad, entendida como el grado de unidad del proceso educativo, aunando los distintos ámbitos cognitivo-afectivo, motriz, de actitud, en la formación de la personalidad de los niños y en la organización y funcionamiento de grupos sociales. La atención se ha de centrar en la persona, a partir de su acción motriz, actuando y comprometiéndose en la actividad físico-deportiva, a la vez que se recrea y desarrolla relaciones de convivencia con sus coetáneos. De tal modo que, según Ríos (2006), para llevar a cabo el deporte adaptado a las personas con discapacidad es imprescindible conocer qué modalidades pueden llegar a ser practicadas teniendo éxito en el contexto escolar.

En este documento se describe la gratificante experiencia de unas jornadas lúdico-deportivo-recreativas llevada a cabo con alumnos videntes e invidentes, en el albergue juvenil de la ciudad de Cazorla (Jaén), con el claro objetivo de mejorar las habilidades y el desarrollo de los niños, así como fomentar la integración de los invidentes en una sociedad cada vez más sensibilizada. Ha sido un espacio de encuentro con un componente lúdico muy importante, así como de relación, convivencia y de participación deportiva, que ha buscado neutralizar el aislamiento que supone el estar escolarizados los niños invidentes en centros ordinarios muy separados entre sí, conteniendo actividades de conocimien- to y aprendizaje de prácticas deportivas, así como otras de carácter cultural, lúdico y relacional.

\section{Plan de actuación.}

Dentro de este apartado se incide sobre la programación semanal que se llevó a cabo con el grupo de niños, se describe cada una de las actividades físico deportivas, en el medio natural y los juegos que se pusieron en práctica durante la semana de convivencia, además de incluir el listado del material necesario para poder realizarlos. Las distintas actividades contaron con la ayuda de cuatro monitores, que tuvieron a su cargo el grupo de niños formado por seis varones y cuatro mujeres de edades comprendidas entre los 10 y 16 años. De los componentes del grupo, hay que resaltar que la gran mayoría tenían una deficiencia visual con restos visuales, excepto uno que tenía una ceguera total y dos de ellos que eran familiares directos.

Para la realización de las actividades lúdico-deportivo-recreativas, los monitores contaron con las propias instalaciones del albergue que eran una pista polideportiva de 20x40 metros, piscina de verano de 25 metros, sala de usos múltiples, sala de juegos y sala de televisión.

\subsection{Programación semanal.}

En este apartado se detalla de manera pormenorizada el programa de actividades planificado a lo largo de toda la semana desde la llegada al albergue el lunes por la mañana hasta la salida el domingo para estar de vuelta al mediodía en sus respectivos hogares, para lo cual hemos considerado oportuno incluir el plan semanal (anexo 1).

\subsection{Descripción de actividades.}

Si por las limitaciones del juego es inviable la participación de algún chico con discapacidad, podrá practicar en pequeño grupo un deporte adaptado (Ríos, 2006). Sus compañeros simularán la discapacidad (si ello es posible o/y necesario) conociendo así otras modalidades deportivas. Para facilitar las interacciones entre los alumnos con discapacidad, según Ríos (2003), se ha de optar por la figura del educador como compañero de juegos, lo que facilita el seguimiento individual de cada alumno en los talleres, garantizando la interrelación entre los participantes discapacitados y aumentando la interrelación entre los niños y los educadores.

\subsubsection{Actividades físico-deportivas.}

- Gymkhana: el grupo de 10 alumnos, dividido en dos equipos (ambos formados por invidentes y videntes) realizaron una serie de pruebas que estaban repartidas por todo el albergue. Para poder pasar a la siguiente prueba debían reconocer el objeto que se les iba dando, realizando esta última acción con los antifaces puestos (anexo 2: Planilla de Gymkhana).

- Cross de Orientación: el grupo de 10 alumnos, dividido nuevamente en dos equipos (ambos formados por invidentes y videntes) realizaron un cross de orientación por las instalaciones del albergue. A cada equipo se le daba un mapa que tenían que descifrar para poder realizar el recorrido correctamente. Durante el recorrido debían responder las preguntas que se les hacía acerca del recorrido. Para la elaboración tanto del recorrido como del mapa de orientación, se tuvieron en cuenta las pautas y premisas marcadas por autores como García Montes y Hernández Rodríguez (1998) y Bocanegra y Villanueva (2003) (anexo 3: Mapa Cross de Orientación).

- Taller de Goalball: el Goalball es un deporte específicamente paralímpico practicado por personas ciegas y deficientes visuales, que enfrenta a dos equipos de tres jugadores. Los partidos constan de dos tiempos de diez minutos cada uno, y la pista tiene que tener unas medidas de 9 por 18 metros. Cada equipo se sitúa a un lado del campo junto a su portería de 9 metros de ancho y, sin salirse de una pequeña zona, tiene que tratar de meter gol en la portería contraria lanzando el 


\begin{tabular}{|c|c|c|c|c|c|c|c|}
\hline & LUNES & MARTES & MIERCOLES & JUEVES & VIERNES & SABADO & DOMINGO \\
\hline $\begin{array}{l}8: 30 \\
9: 00\end{array}$ & \multirow[b]{3}{*}{ Viaje de ida } & Diana & Diana & Diana & Diana & Diana & Diana \\
\hline $\begin{array}{c}9: 00 \\
10: 00\end{array}$ & & Desayuno & Desayuno & Desayuno & Desayuno & Desayuno & Desayuno \\
\hline $\begin{array}{l}10: 00 \\
12: 45\end{array}$ & & $\begin{array}{l}\text { Taller de } \\
\text { Goalball }\end{array}$ & $\begin{array}{c}\text { Senderismo: } \\
\text { Visita Castillo } \\
\text { de la Yedra y } \\
\text { Centro de } \\
\text { crianza de } \\
\text { Aves }\end{array}$ & $\begin{array}{c}\text { Taller de } \\
\text { Atletismo y } \\
\text { Fútbol Sala }\end{array}$ & $\begin{array}{c}\text { Senderismo: } \\
\text { Visita Castillo } \\
\text { de Iruela y } \\
\text { Virgen de la } \\
\text { Cabeza }\end{array}$ & $\begin{array}{c}\text { Taller de } \\
\text { juegos }\end{array}$ & \multirow[t]{2}{*}{$\begin{array}{l}\text { Viaje de } \\
\text { vuelta }\end{array}$} \\
\hline $\begin{array}{l}12: 45 \\
14: 00\end{array}$ & $\begin{array}{c}\text { Reconocimien } \\
\text { to del } \\
\text { albergue }\end{array}$ & $\begin{array}{c}\text { Taller de } \\
\text { Natación } \\
\text { Terapéutica }\end{array}$ & $\begin{array}{c}\text { Juegos de } \\
\text { Piscina }\end{array}$ & $\begin{array}{c}\text { Taller de } \\
\text { Natación } \\
\text { Terapéutica }\end{array}$ & $\begin{array}{c}\text { Juegos de } \\
\text { Piscina }\end{array}$ & $\begin{array}{l}\text { Natación } \\
\text { Terapéutica }\end{array}$ & \\
\hline $\begin{array}{l}14: 00 \\
15: 00\end{array}$ & Almuerzo & Almuerzo & Almuerzo & Almuerzo & Almuerzo & Almuerzo & \\
\hline $\begin{array}{l}15: 00 \\
17: 00\end{array}$ & Tiempo Libre & Tiempo libre & Tiempo libre & Tiempo libre & Tiempo libre & Tiempo libre & \\
\hline $\begin{array}{l}17: 00 \\
18: 30\end{array}$ & $\begin{array}{c}\text { Taller de } \\
\text { Natación } \\
\text { Terapéutica }\end{array}$ & $\begin{array}{c}\text { Juegos de } \\
\text { piscina }\end{array}$ & $\begin{array}{c}\text { Taller de } \\
\text { Natación } \\
\text { Terapéutica }\end{array}$ & $\begin{array}{c}\text { Juegos de } \\
\text { piscina }\end{array}$ & $\begin{array}{c}\text { Taller de } \\
\text { Natación } \\
\text { Terapéutica }\end{array}$ & $\begin{array}{c}\text { Juegos de } \\
\text { piscina }\end{array}$ & \\
\hline $\begin{array}{l}18: 30 \\
20: 00\end{array}$ & $\begin{array}{l}\text { Taller de } \\
\text { Goalball }\end{array}$ & $\begin{array}{c}\text { Taller de } \\
\text { Atletismo }\end{array}$ & $\begin{array}{c}\text { Cross de } \\
\text { Orientación }\end{array}$ & Gymkhana & $\begin{array}{l}\text { Taller de } \\
\text { Goalball }\end{array}$ & $\begin{array}{c}\text { Visita pueblo: } \\
\text { Compra de } \\
\text { Souvenir }\end{array}$ & \\
\hline $\begin{array}{l}20: 00 \\
20: 45\end{array}$ & Aseo personal & Aseo personal & Aseo personal & Aseo personal & Aseo personal & Aseo personal & \\
\hline $\begin{array}{l}20: 45 \\
21: 30\end{array}$ & Cena & Cena & Cena & Cena & Cena & Cena & \\
\hline $\begin{array}{l}21: 30 \\
23: 45\end{array}$ & $\begin{array}{c}\text { Visita pueblo } \\
\text { y Juegos de } \\
\text { Mesa }\end{array}$ & $\begin{array}{c}\text { Juegos en la } \\
\text { plaza del } \\
\text { pueblo y } \\
\text { Taller de } \\
\text { Relajación }\end{array}$ & $\begin{array}{c}\text { Visita al } \\
\text { pueblo y } \\
\text { Juegos de } \\
\text { mesa }\end{array}$ & $\begin{array}{c}\text { Juegos en el } \\
\text { parque } \\
\text { principal y } \\
\text { Cuenta } \\
\text { cuentos }\end{array}$ & $\begin{array}{l}\text { Visita pueblo } \\
\text { y Taller de } \\
\text { relajación }\end{array}$ & $\begin{array}{c}\text { Fiesta de } \\
\text { despedida }\end{array}$ & \\
\hline $\begin{array}{l}23: 45 \\
24: 00\end{array}$ & Silencio & Silencio & Silencio & Silencio & Silencio & Silencio & \\
\hline
\end{tabular}

\section{Anexo 1. Plan semanal}

balón con cascabeles en su interior, que ha de rodar por el suelo para que se oiga su sonido. Los tres componentes del equipo que recibe el balón tienen que impedir que entre en su portería arrojándose al suelo para detenerlo. Todos los jugadores han de salir a la cancha con unos antifaces opacos que les impiden ver absolutamente nada, garantizando así la igualdad de condiciones entre ciegos totales y deficientes visuales (Fernández, 2002a).

Este taller se llevó a cabo en la pista polideportiva del albergue adaptándola a las necesidades específicas de la actividad a realizar. Durante el desarrollo de la actividad observamos y experimentamos las dificultades de este deporte como pueden ser la orientación en el espacio, la necesidad de silencio absoluto, trabajo en equipo... pese a ello la actividad se llevó a cabo perfectamente.

- Taller de Fútbol Sala: la iniciación al fútbol sala fue la más complicada de llevar a cabo, ya que había que desplazarse por el espacio con el balón o sin él, aspecto que muy pocos lo dominaban por la dificultades propias de las discapacidades visuales, así que nos centramos en que aprendiesen a controlar la pelota y orientarse en el espacio de la pista de fútbol sala lo mejor posible, sintiendo así las sensaciones propias del fútbol sala para invidentes.

- Taller de Natación Terapéutica: la práctica de actividades físicas en el agua, se puede realizar en cualquier posición en el espacio, casi sin limitaciones, amentando así la riqueza de movimientos, mejorando el esquema corporal, el conocimiento del cuerpo en el espacio y el desarro- llo de la orientación espacial (Fernández, 2002a). Este taller consistió en realizar una serie de «ejercicios» orientados a la salud con una finalidad correctivo-preventiva (Lloret, 2004), aprovechando las propiedades físicas que el agua nos brinda. La actividad se llevó a cabo en la piscina del albergue de forma coordinada y dirigida por un monitor y un socorrista.

- Taller de Atletismo: el atletismo es una actividad deportiva que puede facilitar el desarrollo de valores sociales tan importantes como la integración, aportando pautas educativas y de desarrollo físico, psíquicoy social, incidiendo en las habilidades básicas del individuo, desplazamientos, saltos y lanzamientos y mejorando los elementos perceptivocorporales y espacio-temporales, base de la motricidad humana (Valero, 2004).

Prueba de velocidad 60 metros lisos: el grupo de 10 alumnos, agrupados por parejas (invidente-vidente) y unidos mediante una pequeña cuerda, realizaron la carrera de 60 metros en la que en ningún momento pudieron soltarse del compañero o salirse de la calle que les correspondía.

Prueba de salto de longitud: de forma consecutiva fueron saltando cada miembro de la pareja desde una línea (tabla de batida), colocando un pie adelantado y debiendo caer lo más alejado posible de ésta con ambos pies. Se midió la distancia alcanzada desde la línea de batida hasta el punto más cercano a ella con el que se contactaba en la colchoneta (talones, glúteos, manos, espalda...). 
Prueba de salto de altura: de forma consecutiva fueron saltando cada miembro de la pareja situándose de espaldas a la colchoneta y eligiendo la altura a la que deseaban colocar el listón (goma elástica) para superarlo sin ser rozado. En caso de ser contactado se consideraba nulo, disponiendo de otro intento.

Prueba de lanzamiento de peso: de forma consecutiva fueron lanzando cada miembro de la pareja (comenzando el invidente y recogiendo el vidente de la siguiente pareja), no pudiendo aprovechar el espacio del círculo de lanzamiento para realizar un desplazamiento previo. El lanzador tampoco podía abandonar el círculo de lanzamiento por la parte delantera, considerándose nulo y perdiendo la posibilidad de ser medido su tiro. Igualmente fue nulo todo lanzamiento que caía fuera del sector dedicado a tal efecto.

\subsubsection{Actividades en el medio natural.}

Realizamos tres salidas, la primera al Castillo de la Yedra, ruinas de la Iglesia principal del pueblo y Centro de crianza de Aves de Sierra de Cazorla, la segunda al Castillo de Iruela y Virgen de la Cabeza y la tercera al casco antiguo del pueblo. En cada una de las salidas, se desarrollaron visitas culturales acompañadas de explicaciones interactivas por parte del guía donde se explicaba la historia, características y curiosidades de cada uno de los monumentos visitados.

En la primera salida visitamos el Castillo de la Yedra el cual estaba situado en la cima de una montaña cerca del casco antiguo de la ciudad. Por el camino guiamos a los niños que tenían mayores dificultades sin que para ello hubiera ningún contratiempo, transcurriendo todo con total normalidad. En el camino de vuelta al albergue visitamos tanto las ruinas de la Iglesia más antigua e importante del pueblo, como un Centro de Crianza de Aves de la Sierra de Cazorla en el cual nos explicaron en que consistía el trabajo que llevaban a cabo en el centro y las especies de aves que criaban en el.

La segunda salida se destinó a la visita del Castillo de la Iruela y del santuario de la Virgen de la Cabeza, situado el primero a unos cinco kilómetros aproximadamente del albergue y el segundo a ocho. En esta segunda visita, la dificultad fue mayor que la primera, debido a la orografía del terreno y a la distancia de los lugares.

En la tercera salida se visitó el pueblo, abordando los monumentos más representativos (plazas, iglesias, conventos...). Al mismo tiempo, esta salida fue utilizada por la mayoría del alumnado para comprar souvenir a sus familiares.

\subsubsection{Juegos.}

\section{- Juegos de Mesa:}

Parchís: se realizó un campeonato de parchís, el cual estaba adaptado para los invidentes. Mediante una liguilla que jugaban todos contra todos, es decir, videntes e invidentes en las mismas condiciones. A los videntes, se les colocaba un antifaz, haciendo el juego más igualitario y haciéndoles sentir a estos, las sensaciones de jugar sin ver nada. Antes de iniciar el campeonato tuvimos que enseñar a jugar al parchís a un invidente, el cual aprendió rápidamente las reglas y el objetivo del juego. Casualmente fue ese niño el que ganó el campeonato.

Dominó: en este juego de mesa también se realizó un campeonato, pero esta vez por parejas formadas por vidente-invidente. El campeonato tenía las mismas características que el de parchís, es decir, una liguilla. Las piezas del dominó estaban adaptadas para los invidentes y las leían contando el número de agujeros que había en cada ficha.

- Juegos desarrollados en las pistas Polideportivas: en las pistas situadas dentro del recinto del albergue llevamos a cabo una serie de juegos adaptados a los niños con deficiencia visual (Arráez, 1997; Linares y Arráez, 1999). Los contenidos principales de estos juegos fueron: orientación espacial, percepción auditiva y táctil, habilidades motrices básicas y cooperación.

Por ejemplo uno de los juegos que se llevaron acabo fue «El Arca de Noé» que consistía que cada pareja eligiera el sonido de un animal y se desplazara por el espacio imitando el sonido del animal y su forma de desplazamiento (cuadrupedia, saltos...). Este desplazamiento se realizaba con los ojos tapados (con antifaces en los casos que hacía falta) por todo el espacio. A la señal del monitor, los miembros de cada pareja debían buscarse mediante los sonidos del animal que escogieron y una vez que se localizaban debían abrazarse.

- Juegos desarrollados en plazas y parques del pueblo: En el parque y plazas del pueblo se llevaron a cabo dos tipos de juegos. En primer lugar, juegos libres, es decir, los niños jugaban en las instalaciones de forma libre, subiéndose en toboganes, columpios, etc. y en segundo lugar, juegos organizados y controlados por los monitores, entre los cuales podemos resaltar los juegos populares y tradicionales.

- Juegos desarrollados en la piscina: los juegos que se realizaron fueron múltiples y variados (Moreno Murcia, 2001), supervisados en todo momento por personas responsables, en este caso, por los diferentes monitores y el socorrista de la piscina. Teniendo en cuenta todos los juegos realizados y el medio en el que se llevaron a cabo fue una de las actividades más exitosas y que más les gustó a los niños.

-Tallerde Relajación: La relajación contribuye a combatir lahipertonía muscular que estos sujetos desarrollan como consecuencia del miedo a lo desconocido, a los golpes y a las caídas (Arráez, 1998). Este taller se llevó a cabo dos noches con el fin de que los niños experimentasen y aprendiesen una iniciación a la relajación mediante unos ejercicios muy sencillos, basados en una serie de movimientos lentos, pausados y coordinados sintiendo todas las partes del cuerpo, en dirección céfalocaudal y próximo-distal.

\subsubsection{Tiempo libre.}

En las horas que disponían de tiempo libre realizaban las actividades que más les gustaban tales como: bañarse en la piscina, ver la televisión, jugar al dominó o al parchís, descansar, leer, conversar con los compañeros...

\subsection{Material necesario.}

Dentro del siguiente apartado realizamos un listado del material que fue empleado para poner en marcha cada una de las actividades que fueron programadas para las jornadas lúdico-deportivo-recreativas.

\subsubsection{Taller de fútbol sala y goalball.}

- Una pelota específica para cada uno de los deportes, una de Goalball y otra para Fútbol Sala (ambas pelotas contienen cascabeles en su interior).

- Un par de rodilleras y coderas por jugador.

- Diez antifaces deportivos o gafas de natación que impidan la entrada de la luz.

- Diez protectores para la cabeza.

- 2 rollos de cinta aislante.

- Cuerdas.

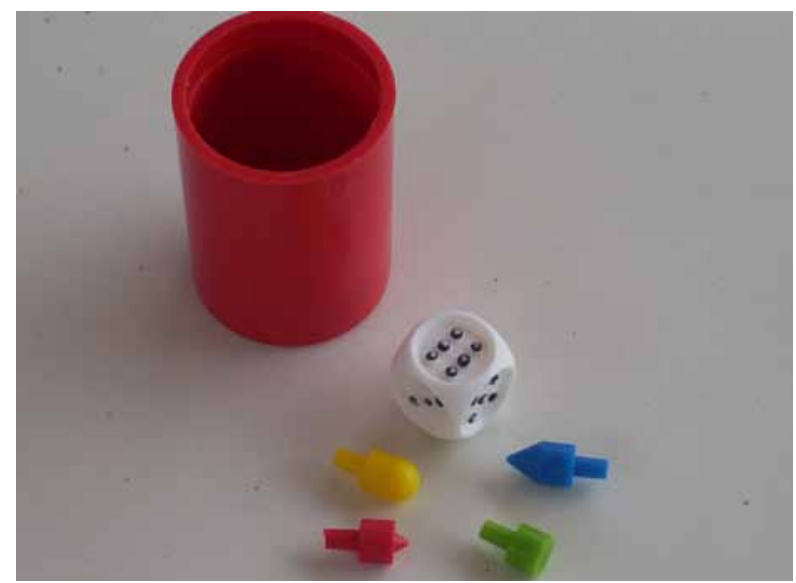




\subsubsection{Taller de Natación Terapéutica.}

- Flotadores en forma de chalecos.

\subsubsection{Taller de atletismo.}

- Cinta métrica.

- Balón medicinal de bajo peso (aproximadamente 2 kg.).

- Cinco aros o cuerdas pequeñas.

- Colchonetas finas y quitamiedos.

- Saltómetros.

- Cinta elástica.

\subsubsection{J uegos.}

- Cinco picas.

- Una cuerda grande.

- Cinco cuerdas pequeñas.

- Diez aros planos y grandes.

- Un elástico.

- Cinco petos de color llamativo.

\subsubsection{Taller de relajación.}

- 1 Cassete con cd.

- 1 colchoneta de aeróbic por persona.

\subsubsection{Gymkhana.}

- Plano (anexo 2).

\subsubsection{Cross orientación.}

- Plano (anexo 3).

\subsubsection{Salidas y visitas culturales.}

- Vestimenta adecuada para la práctica del senderismo.

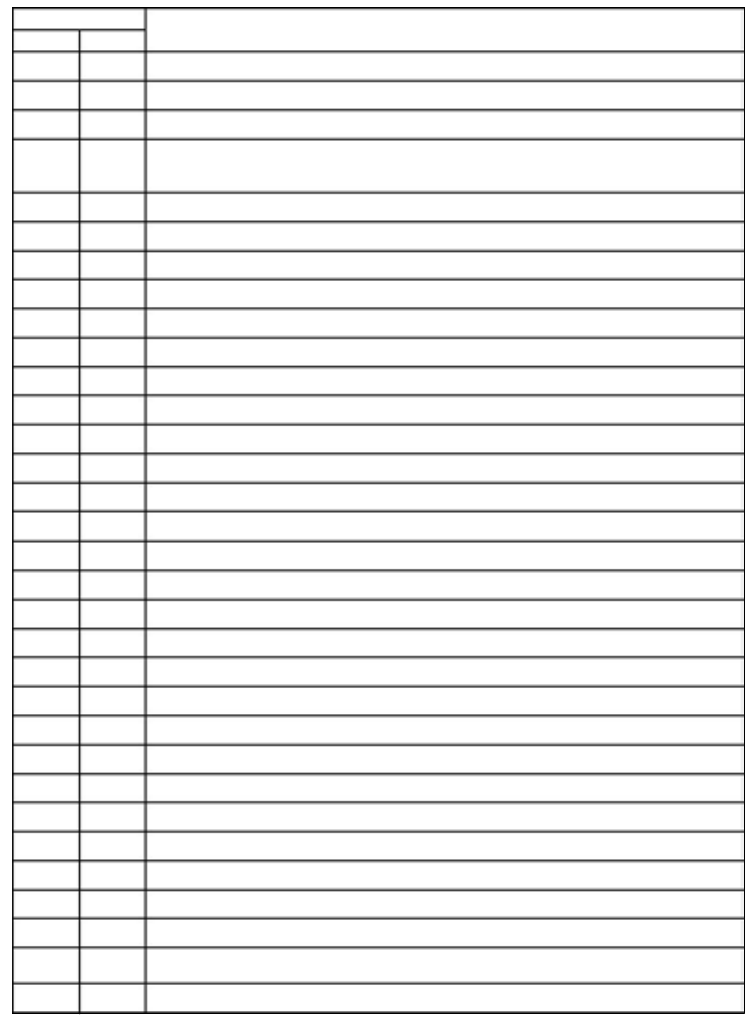

Anexo 2. Plantilla de Gymkhana
- Cantimplora.

- Gorra.

- Crema solar.

- Barra de cacao para los labios.

\section{Conclusiones.}

La promoción de acciones para la atención de las personas con discapacidad, ha de seguir creciendo en importancia en el mundo actual, debido al incremento cuantitativo de las situaciones de discapacidad, así como la toma de conciencia de que es necesario aprovechar las capacidades, los conocimientos y la contribución al desarrollo que pueden hacer las personas con discapacidad.

El disfrute del ocio y tiempo libre es un derecho de todos y a todos nos aporta calidad de vida. Las personas que trabajamos y colaboramos en la recreación con estas personas tenemos que saber que cualquier actividad que se realice dependerá de nuestra actitud y forma de apoyarles el que las disfruten de pleno derecho y con libertad.

Debemos valorar el aprendizaje por encima del rendimiento, para lo cual se han de proponer actividades orientadas al proceso y no al producto, promoviendo así la participación por la participación, poten-

\section{$\underline{\text { ANEXOS }}$}

CROOS DE ORIENTACIÓN

> Guiate por las pistas y los objetos del recorrido y contesta a todas las preguntas

\begin{tabular}{|c|c|c|c|}
\hline $4_{6 . P}$ & $\begin{array}{l}2 \\
\text { ¿QVÉ SE ENCVEMASA } \\
\triangle T V \text { DSRECHA? }\end{array}$ & $4_{8 p}^{3} \rightarrow T^{\text {Toncer }}$ & $\begin{array}{l}4 \\
\text { ¿CVASTASS LVCES } \\
\text { VES EN ESTS } \\
\text { HABIDCIÓN? }\end{array}$ \\
\hline DOMPASE - INEEMOOO & 6 & 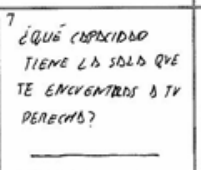 & \\
\hline 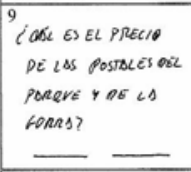 & $\int_{200}^{4}$ & $\begin{array}{l}11 \text { ¿CVÓNTOS ADQRINAS } \\
\text { VES? } \\
\end{array}$ & $\begin{array}{l}\text { icusines cusares } \\
\text { BLANCOS res? }\end{array}$ \\
\hline$\left.\square^{13}\right|^{x}$ & 14 & $\begin{array}{l}15 \text { ¿CUANTAS DVCHOS } \\
\text { HAY EN LS PISEINA? } \\
\text { ¿Y FLOTBDones? }\end{array}$ & $\left.\begin{array}{l}16 \\
\text { lcovomos } \\
\text { Rompas } \\
\text { rat? }\end{array}\right\} \hat{\jmath}$ \\
\hline 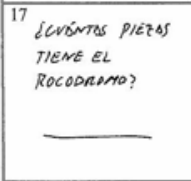 & CaVE ves OTU DERECOAD & 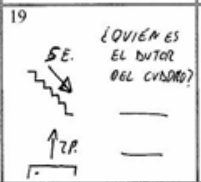 & $\begin{array}{r}20 \text { SALIOD } \\
4\end{array}$ \\
\hline
\end{tabular}

ciando los aspectos lúdicos y la interrelación positiva entre los participantes.

«E ducar para la inclusión es fomentar sentimientos de confianza y respeto basados en el conocimiento. Aprender a no discriminar es aprender a confiar y a respetar a quien es distinto, porque su aspecto externo es diferente del nuestro o porque no piensa y actúa igual que nosotros, sobre todo si se encuentra en una posición de inferioridad o debilidad. Sólo sepuedetener respeto y confianza en alguien a quien se conoce. El resultado final de la educación para la inclusión debeser el compromiso personal quecada cual contraea favor delosderechos de los demás» (Fernández y cols., 2002a).

No es preciso utilizar deportes estandarizados y regulados para hacer deporte inclusivo, ya que lo que es verdaderamente importante es la adaptación de actividades físico-deportivas corrientes a las diversas 


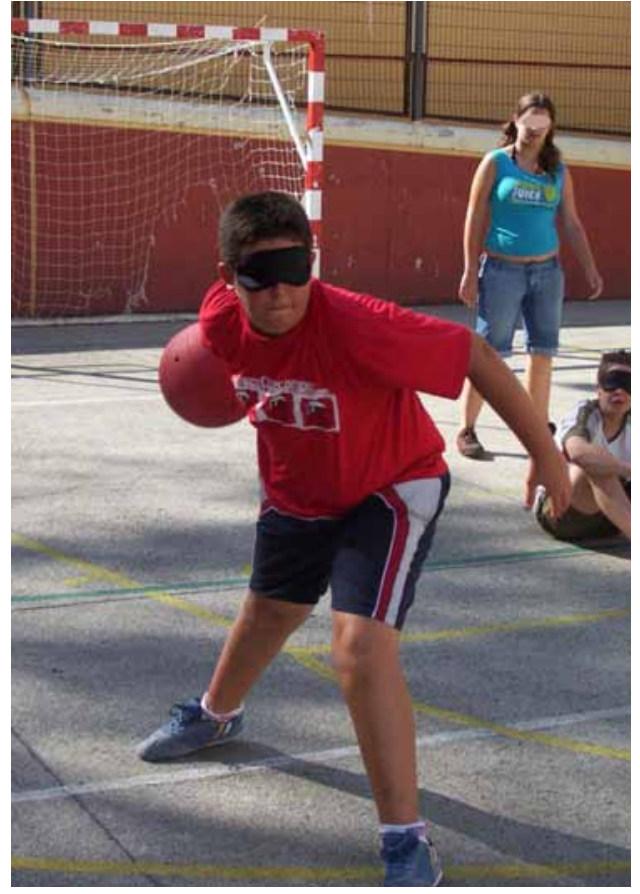

limitaciones, creando así situaciones lúdicas y divertidas donde las diferencias existentes, es decir la falta de visión, sea la primera regla del juego.

Con el fin de generar un cambio en el acercamiento de estos jóvenes con discapacidad visual a la práctica físico-deportiva es indispensable contar con personal capacitado, concienciar a los padres, docentes y responsables de la importancia que tiene la práctica física lúdica y recreativa, ya que si conseguimos que por ejemplo los padres valoren el trabajo que hacemos con sus hijos, seguramente habremos logrado que éstos potencien esta práctica físico-deportiva en ellos, lo cual es fundamental debido a la influencia que los padres ejercen en los niños, y más aun, aquellos con necesidades especiales.

Debemos considerar la reflexión como el elemento más importante, pues es en ella en la que en mayor medida se cambian actitudes y adquieren conceptos, no debiendo reducir estas actividades en mero activismo, ya que no estaríamos necesariamente educando, si no viene acompañada de un trabajo de la capacidad reflexiva (Fernández y cols., 2002b).

Finalmente, estas jornadas han contribuido a mejorar el desarrollo integral de los chicos tanto videntes como invidentes, al tiempo que se han sensibilizado niños participantes, responsables de las actividades y personas del entorno sobre la problemática del mundo del discapacitado, haciéndoles ver la importancia que tiene su contribución por pequeña que pueda parecerles, pues supone una gran ayuda para ellos.

\section{Bibliografía.}

Arráez, J. M. (1997). ¿Puedo jugar yo?. Granada: Proyecto Sur. Arráez, J. M. (1998). Teoría y praxis de las adaptaciones curriculares en la Educación Física. Un programa de intervención motriz aplicado en la Educación Primaria. Málaga: Aljibe.

Barrado, J. M. (2004). La actividad física y deportiva de las personas ciegas y deficientes visuales. En M. A. González, J. A. Sánchez y J. Gómez (Ed.). Congreso Internacional de la AIESEP. Preparación profesional ynecesidades sociales (35-40). ACoruña: Instituto Nacional de Educación Física. Universidade da Coruña.

Bocanegra, C. y Villanueva, A. (2003). Pautas para la elaboración de mapas de orientación de centros escolares y de jardines. Retos, Nuevas Tendencias en Educación Física, Deportey Recreación, 6, 21-25.

Cuadrado, G., Mendoza, N. y Pérez, R. (2001). Influencia de la práctica de actividad física en los aspectos físicos del parapléjico sedentario. Apunts, Educación Física y Deportes, 66, 54-59.
Duque, C. y Mosquera, J. (2004). La importancia de la implementación de actividades recreativas, para el manejo del tiempo libre de las personas con limitación visual. En VIII Congreso Nacional de Recreación. Bogotá: Coldeportes. Funlibre. (En línea). http::// www.redcreacion.org/documentos/congreso8/CDuque.htlm. (consulta: 17 noviembre 2006).

García Montes, M. E. y Hernández Rodríguez, A. I. (1998). Recorridos de orientación urbana: una forma de apropiarse de la ciudad. Espacio y Tiempo, 22-23-24, 55-69.

Fernández, C., Diego, T. Vaquero, J. L. Alonso, G. Barrado, J. M., Añino, S., Cofre, A.,

García, J. A. y Villagra, A. (2002a). Unidad Didáctica. Deporte sin exclusiones. Libro del alumno. Madrid: CSD.

Fernández, C., Diego, T. Vaquero, J. L. Alonso, G. Barrado, J. M., Añino, S., Cofre, A., García, J. A. y Villagra, A. (2002b). Unidad Didáctica. Deporte sin exclusiones. Libro del profesor. Madrid: CSD.

Gómez, M. (2005). La actividad físico-deportiva en los centros almerienses de Educación Secundaria post obligatoria y en la Universidad de Almería. Evolución de los hábitos físico-deportivos de su alumnado. Universidad de Almería: Servicio de Publicaciones.

Linares, P. (1997). Motricidad en un grupo de personas con necesidades educativas especiales. Motricidad, 3, 187-207.

Linares, P. y Arráez, J. M. (1999). Motricidad y necesidades especiales. Granada: AEMNE.

Loeckemeyer, M. (2006). Acercamiento del discapacitado visual al deporte. Porqué y para qué? Comunidad Deportiva (1), 1-3. (En línea). http://www.deportes.gov.ar/caid/Plug/Notas.php?Ver=7 (consulta: 17 noviembre 2006).

López Rodríguez, A. y Moreno Murcia, J. A. (2000). Integralidad, variabilidad y diversidad en educación física. Lecturas: Educación Física y Deportes, Revista Digital (19), 1-20. (En línea). http::// www.efdeportes.com/efd30/discap.htm. (consulta: 17 noviembre 2006).

Lloret, M. (2004). Natación terapéutica. Barcelona: Paidotribo.

Moreno Murcia, J. A. (2001). Juegos acuáticos educativos. Barcelona: Inde.

Ortiz, J. D. (2001). Iniciación a los deportes de aventura para discapacitados sensoriales visuales. Lecturas: Educación Físicay Deportes, Revista Digital (30), 1-16. (En línea). http::// www.efdeportes.com/efd81/atl.htm. (consulta: 17 noviembre2006).

Ríos, M. (2003). El juego y el alumnado con discapacidad intelectual y/ o plurideficiencia. Tándem, Didáctica de la Educación Física, 11, 40-49.

Ríos, M. (2006). Estrategias inclusivas en el área de Educación Física. Tándem, Didáctica de la Educación Física, 21, 81-91.

Ruiz Juan, F. y García Montes, M. E. (2005). Hábitos físico-deportivos de los almerienses en su tiempo libre. Universidad de Almería: Servicio de Publicaciones.

Ruiz Juan, F., García Montes, M. E. y Pérez, A. (2005). Estilos de vida en Ciudad de la Habana-Cuba. Hábitos físico-deportivos y de salud. Madrid: Gymnos.

Santana, A. y Guillén, F. (2002). Intereses y actitudes de las personas ciegas o con deficiencia visual por la actividad físico-deportiva, según la edad y el sexo. Apunts, Educación Física y Deportes, 69, 76-83.

Valero, A. (2004). Situación actual de la metodología en la iniciación deportiva al atletismo. Revista de Educación Física: Renovar la teoría y la práctica, 94, 13-20.

Valero, A. y Gómez, M. (2005). Propuesta práctica de un taller de atletismo para la iniciación deportiva en niños con discapacidad visual. Lecturas: Educación Física y Deportes, Revista Digital (81), 1-13. (En línea). http:://www.efdeportes.com/efd81/atl.htm. (consulta: 17 noviembre 2006). 